\title{
Analytical Approximation for the Inductance of Circularly Cylindrical Two-Wire Transmission Lines with Proximity Effect
}

\author{
H. A. Aebischer ${ }^{1}$, H. Friedli² \\ ${ }^{1}$ LEGIC ${ }^{\circledR}$ Identsystems AG, Switzerland \\ ${ }^{2}$ Formerly Bern University of Applied Sciences, Switzerland \\ *corresponding author, E-mail: hubert.aebischer@legic.com
}

Very sadly, Dr. Hansjörg Friedli passed away before the result of our common research work could be written up in the present paper. The main author wishes to dedicate it to the memory of a gifted physicist and dear friend.

\begin{abstract}
The paper describes a simple analytical approximation for the inductance of two-wire transmission lines of circularly cylindrical wires with proximity effect. It yields precise results up to very high frequencies and at all interaxial distances between the wires above some limit. Its accuracy is established by comparison to numerical computations and to measurements. It is shown that the proximity effect cannot be neglected unless the interaxial distance between the wires amounts to at least four wire diameters. Further, figures of the current distribution in various situations are discussed.
\end{abstract}

\section{Introduction}

At high frequencies inductance decreases compared to its value at DC (direct current) due to skin effect, which has been well documented for wires of circular, annular, and rectangular cross section [1] - [6].

In a two-wire transmission line, the inductance is further reduced by the proximity effect ([3], p. 304-306). Kennelly et al. presented results of precise inductance and resistance measurements on rectangular loop coils composed of circularly cylindrical copper wires at various wire distances and frequencies [5]. Some of these measurements entailed the proximity effect. For simplicity, we will henceforth just write cylindrical instead of circularly cylindrical. Amazingly, despite the importance of the cylindrical two-wire transmission line in radio frequency engineering, the literature on inductance calculation taking the proximity effect into account seems rather scarce. Tsuk and Kong compared Kennelly's results with their numerical calculations, which were based on a combination of the "finite filament method" with an integral equation approach [7]. To apply the finite filament method to conductors of circular cross section, they divided the wires into filaments of triangular cross section instead of the usual rectangular one, and they derived an analytical expression for the GMD (geometric mean distance) between triangles [7]. Carson gave a series solution for the resistance [8]. Wang derived a series representation for the internal partial self-inductance of the two wires [9]. But to be able to compute the total inductance, one also needs expressions for the external and the mutual inductance, which he didn't provide.

The conclusion is that, as of today, calculating inductance considering the proximity effect remains an ambitious task. There seems to be no way around extensive numerical calculations. For engineering purposes it would be very helpful to have a simple calculational method at hand. Further, it even seems that no systematic calculations covering a comprehensive range of wire distances and frequencies have yet been reported. Also, we couldn't find any figures of current distribution in the literature.

In this paper we intend to fill these gaps. We describe a simple yet precise analytical approximation for the inductance of a cylindrical two-wire transmission line that takes the proximity effect into account. We verify it by means of systematic numerical calculations for a set of interaxial wire distances and frequencies. In section 2 we describe the numerical method used. In section 3 we report the results obtained, and we also discuss figures of the current distribution in various situations. Section 4 introduces our analytical approximation and tests it against the numerical results. In section 5 we compare it to measurements reported in the literature and to own measurements. Conclusions are given in section 6 .

\section{Numerical solution method}

In its original form, the "finite filament method" is based on the cross-sectional subdivision of the conductor into many equal filaments of small but finite rectangular cross section, all the same length as the conductor [3], [10]. Obviously, the method is perfectly suited for conductors of rectangular cross section. The conductor is modelled as a parallel connection of magnetically coupled filaments. Thus, the potential differences along all filaments are prescribed to be identical. The distribution of current within a filament is assumed to be uniform. But the magnitude of the current varies from filament to filament according to the current distribution in the wire cross section resulting from the calculation, which is subject to the skin and proximity effect caused by the coupling between the filaments. The solution 
is found by solving a system of linear equations involving a huge matrix of impedances. Its diagonal elements are the impedances of the filaments formed by their partial selfinductance and resistance. Its off-diagonal elements account for the mutual magnetic coupling between any two of the filaments. The method relies on the fact that the selfinductance of and the mutual inductance between the filaments can be calculated analytically. One way to do this is to use an analytical expression for the GMD between the rectangular cross sections of the filaments [3], [10].

Tsuk and Kong stated that "Since the method is limited to rectangular elements, it is not capable of handling this case" (i.e. the conductor of circular cross section, [7], p. 1343). We show this statement to be wrong. The finite filament method using filaments of square cross section can well be applied to cylindrical wires. As an example, we mention the calculation of the partial inductance of a single cylindrical wire with skin effect. The application of the method is simple: The circular cross section is embedded in a square, which is divided into $N \times N$ subsquares. Only those subsquares whose central point lies within the cross section of the wire are selected to represent a filament. Amazingly, with only $7 \times 7$ subsquares, the inductance of a wire of $0.5 \mathrm{~mm}$ radius at a frequency of $2 \mathrm{MHZ}$ (the radius is more than 10 times larger than the skin depth) obtained by this method is accurate to $1 \%$ (if the length is at least 25 times larger than the radius).

Unfortunately, the experience gained with single wires with skin effect cannot be transferred to two-wire transmission lines with proximity effect. The latter require much larger numbers of filaments, as we shall see. This is a result of the break-up of circular symmetry in the current distribution with proximity effect. The inductance $L$ depends on four parameters (apart from the temperature and the wire material): the length $l$ of the transmission line, the radius $R$ of the wires, their interaxial distance $d$, and the frequency $f$. We can reduce the dimensionality of the wanted fitting function for the analytical approximation to two by defining normalized parameters which depend only on the relative interaxial distance and the relative wire radius instead of the absolute ones. If the two arguments of the wanted fitting function are relative size parameters, then the approximation will be valid for any size of the transmission line. First, we define the relative interaxial wire distance $\kappa$ by normalizing the absolute distance $d$ to the radius $R$ :

$$
\kappa=\frac{d}{R}
$$

We adopt the natural definition of the relative wire radius or normalized frequency parameter $\zeta$ (proportional to the square root of the frequency $f$ ) as the ratio of the radius to the skin depth $\delta$ :

$$
\zeta=\frac{R}{\delta} \propto \sqrt{f}
$$

with the skin depth

$$
\delta=\sqrt{\frac{1}{\mu_{0} \sigma \pi f}}
$$

where $\mu_{0}$ is the magnetic permeability of the vacuum, $\mu_{0}=$ $4 \pi \cdot 10^{-7} \mathrm{Vs} /(\mathrm{Am})$, and $\sigma$ is the conductivity of the wire material. In our finite filament calculations, we used $\sigma=$ $5.9595 \cdot 10^{7} \Omega^{-1} \mathrm{~m}^{-1}$, corresponding to the resistivity $\rho=$ $1 / \sigma=1.678 \cdot 10^{-8} \Omega \mathrm{m}$ of copper at $20^{\circ} \mathrm{C}([11], T=$ $293 \mathrm{~K})$.

At last, we don't want to approximate the inductance $L$ directly because it still depends on a third variable besides $\kappa$ and $\zeta$, namely, the length $l$. So, we also need to normalize the inductance $L$ somehow to get rid of this third variable, to keep the target function to be fitted two-dimensional. In principle, we could just take the inductance per unit length since the inductance of two-wire transmission lines is proportional to their length, at least if $l \gg d$. But there is a better choice: the normalization to the inductance $L_{\text {skin }}$, in which only the skin effect is considered. Since $L_{\text {skin }}$ is also proportional to $l$, the dependency on $l$ cancels out. Only then did we succeed in finding a simple fitting function when the dependencies on $\kappa$ and $\zeta$ due to the skin effect alone had already been normalized out by means of $L_{\text {skin }}$. This choice of the ratio $L / L_{\text {skin }}$ as the target quantity to be fitted is further justified by the fact that $L_{\text {skin }}$ can easily be calculated analytically. It is given by

$$
L_{\text {skin }}=2\left(L_{1 \text { skin }}-M_{12 s k i n}\right) \text {, }
$$

where $L_{1 \text { skin }}$ is the partial self-inductance of a single wire including skin effect only. For $l \gg R$ it is given by ([12], equation (29) multiplied by $l$, with equation (31) added),

$$
L_{1 \text { skin }}=\frac{\mu_{0} l}{2 \pi}\left\{\log \left(\frac{2 l}{R}\right)-1-\operatorname{Im}\left[\frac{i}{k R} \frac{\mathrm{J}_{0}(k R)}{\mathrm{J}_{1}(k R)}\right]\right\},
$$

where $k$ is the wave number within the wire,

$$
k=\frac{1-i}{\delta}
$$

with $i$ being the imaginary unit, Im denoting the imaginary part, $\mathrm{J}_{0}$ and $\mathrm{J}_{1}$ the (complex) Bessel functions of first kind and orders 0 and 1, respectively, and where log refers to the natural logarithm, as throughout the paper. The quantity $M_{12 \text { skin }}$ in equation (4) is the mutual inductance between the two wires with skin effect only. Due to the circularly symmetric current distribution when there is only skin effect, the mutual inductance may be well approximated by the formula for the mutual inductance of two parallel filaments (of infinitely small cross section), of length $l$ and mutual distance $d$ ([13], equation (12))

$$
\begin{gathered}
M_{12 \text { skin }}=\frac{\mu_{0}}{2 \pi}\left[l \log \left(\sqrt{l^{2}+d^{2}}+l\right)-l \log \mathrm{d}-\sqrt{l^{2}+d^{2}}\right. \\
+d] .
\end{gathered}
$$


In textbooks it is usually assumed that $l \gg d$. Substituting equations (5) and (7) into equation (4) and exploiting that assumption, we find

$$
L_{\text {skin }} \cong \frac{\mu_{0} l}{\pi}\left\{\log \left(\frac{d}{R}\right)-\operatorname{Im}\left[\frac{i}{k R} \frac{\mathrm{J}_{0}(k R)}{\mathrm{J}_{1}(k R)}\right]\right\},
$$

which, in the DC limit $k \rightarrow 0$, yields the well-known formula

$$
L_{D C} \cong \frac{\mu_{0} l}{\pi}\left[\log \left(\frac{d}{R}\right)+\frac{1}{4}\right] \text {. }
$$

In equations (8) and (9), the proportionality of the inductance to $l$ is explicitly visible. But it is better not to rely on approximation (8) and to use the more precise equations (4) - (7) instead. In the evaluation of our own measurements in section 5 , where $l \geq 22 \cdot d$ after all, the approximation (8) would lead to an error of $5 \%$ in $L_{s k i n}$ and, consequently, also in $L$. For even shorter relative lengths, equation (5) and, eventually, equation (7) should be modified to include also other mean distances besides the GMD ([14], equations (33) and (62), respectively).

We wanted to compute the ratio $L / L_{\text {skin }}$ for a dense enough set of parameters $\kappa$ and $\zeta$ allowing us to precisely fit and interpolate the data with a smooth two-dimensional function, and we wanted the domain of this function to be as large as possible. We chose the lower limit $\kappa=2.05$. Smaller values closer to the physical limit $\kappa=2$ might be of interest, but they would have led to computations exceeding the resources available to us. Smaller $\kappa$-values demand bigger resources. With increasing $\kappa$ the proximity effect drops quickly. Beyond $\kappa \geq 10$ it has decreased to the extent that the two wires can be regarded as separated, i.e. only the skin effect remains, at least as far as the inductance is concerned. So, there is no need to go beyond $\kappa=10$. Parameter $\zeta$ cannot be made zero because in equation (5) we have $k$ in the denominator, which becomes zero when $\zeta$ is zero. We chose the lower limit $\zeta=0.05$. This corresponds to $42.5 \mathrm{~Hz}$ for $R=0.5 \mathrm{~mm}$ and to a skin depth of $10 \mathrm{~mm}$. It represents the DC case very well. The upper limit $\zeta=100$ was again dictated by the limits of our computational resources. So, we chose the following 16 values of the wire distance parameter $\kappa: \kappa=2.05,2.1,2.2,2.3,2.4,2.5,2.75$, $3,3.5,4,5,6,7,8,9,10$, and the following 48 values of the wire radius or frequency parameter $\zeta: \zeta=0.05,0.1,0.2$, $0.3,0.4,0.5,0.75,1,1.25,1.5,1.75,2,2.25,2.5,2.75,3,3.25$, $3.5,3.75,4,4.5,5,5.5,6,7,8,10,12,14,16,18,20,22.5,25$, $27.5,30,32.5,35,37.5,40,45,50,55,60,70,80,90,100$.

It was clear that the higher $\zeta$, the more filaments we needed. But how many do we need exactly? A rule of thumb says that the cross-sectional side length of a filament should not exceed half the skin depth. At $\zeta=100$, we have 100 skin depths along a radius, as per definition (2), or 200 skin depths along a diameter. If we follow the above rule of two filaments per skin depth, we have $N=400$ filaments along a diameter, or $400 \times 400$ subsquares in a square of width one diameter. For two squares for the two wires this results in a total of $320^{\prime} 000$ subsquares, of which $251^{\prime} 324$ have their central point within the wire cross section and are thus chosen as filaments. In the computer code, this would lead to two complex-valued matrices of size $251^{\prime} 324 \times$ $251^{\prime} 324$, using more than 1 TByte of RAM (random access memory) each, which lies far beyond the storing capacity of any PC (personal computer). Moreover, two filaments per skin depth are not even enough, as we shall see! One possible remedy would be to adapt the cross-sectional size of the filaments, so that their number increases towards the surface of the wires.

A simpler method is to select only those subsquares as filaments whose central point lies within some multiple $m$ of the skin depth below the surface. This is possible because beyond that distance from the surface the filament current has nearly vanished. Suppose $x$ and $y$ are the rectangular coordinates of the central axis of a filament, with the origin in the axis of the wire. The radial coordinate of the central axis of the filament is then $r=\sqrt{x^{2}+y^{2}}$. The radial coordinate of a point some multiple $m$ of the skin depth $\delta$ below the surface is $R_{\text {skin }}=R-m \delta$. The condition for a subsquare to be selected as a filament is then simply expressed by $R_{\text {skin }} \leq r \leq R$.

Now we are free to define a reasonable number of filaments per skin depth, $n$, without the storage requirements exploding. To ensure a reasonable precision at low $\zeta$, we introduce a third and last parameter defining the problem size, namely, the minimum number $N_{\min }$ of $N$ to be taken in any case. By assigning numbers to the three parameters $m, n$, and $N_{\text {min }}$, the equivalent number of filaments can be computed. The question now was: How large should these three parameters be? There was no way to calculate them beforehand. All we could do was to start with some values, and then to gradually increase them one by one and see how the plots of the results in function of $\kappa$ and $\zeta$ evolved. We started with the parameter set $\left(m, n, N_{\text {min }}\right)=(1,2,20)$, which led to matrices of size $5^{\prime} 120 \times 5^{\prime} 120$ at $\zeta=100$. Fig. 1 shows the resulting curves of the ratio $L / L_{\text {skin }}$ in function of $\zeta$ for $\kappa=2.05,3.0$, and 10.0 . One clearly sees how the ratio decreases from unity at DC with increasing $\zeta$, the stronger so the closer the wires. In the finite filament method, a result for each of the two physical quantities $L_{1}$, which is the partial self-inductance of one of the wires, and $M_{12}$, which is the mutual inductance between the wires, can be extracted (from equation (6.75) in [3]). Based on $L_{1}$ and $M_{12}$, the final inductance of the transmission line is then calculated as

$$
L=2\left(L_{1}-M_{12}\right)
$$

At DC and/or large wire distances, $L_{1}$ equals $L_{1 \text { skin }}$, and $M_{12}$ equals $M_{12 \text { skin }}$, so that $L / L_{\text {skin }}=1$. When the wires move closer together and the wire radius or the frequency is increased, both inductances decrease and both mutual inductances increase, so that the differences (4) and (10) both decrease. But because of the changing current distribution due to the proximity effect, $M_{12}$ increases faster than $M_{12 \text { skin }}$, so that the difference (10) decreases faster than (4). Therefore, the ratio $L / L_{\text {skin }}$ sinks, see Fig. 1 . 


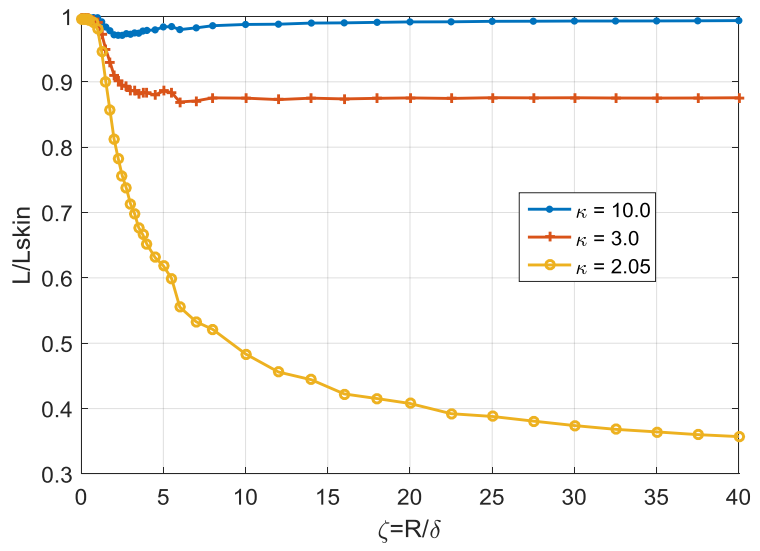

Figure 1: The ratio $L / L_{\text {skin }}$ computed with the parameter set $\left(m, n, N_{\min }\right)=(1,2,20)$ in function of $\zeta$ for $\kappa=$ $10.0,3.0$, and 2.05 , in this order from top to bottom.

But the curves in Fig. 1 are not smooth. They exhibit kinks and other deviations due to lacking numerical precision. The insufficient precision is also revealed by the fact that the curve for $\kappa=10.0$ significantly deviates from its ideal course, which would be a horizontal line close to unity. It turned out that we needed to increase the three parameters $\left(m, n, N_{\text {min }}\right)$ up to the values $(4,5,80)$ before the curves of $L / L_{\text {skin }}$ in function of $\zeta$ got smooth. Despite our sizereducing method, we had to deploy the computations to the external computing cloud EC2 by Amazon ${ }^{\circledR}$, which used Intel ${ }^{\circledR}$ Xeon processors operating at $2.3 \mathrm{GHz}$ and having up to 256 GByte RAM. The machines were designed for parallel processing. The access from within the programming language MATLAB ${ }^{\circledR}$ was provided by The MathWorks ${ }^{\circledR}$ company via the MATLAB Cloud Center using the MATLAB Parallel Computing Toolbox. Since we were the first Swiss customers using the MATLAB Cloud Center [15], we profited from special support from The MathWorks company teams in Switzerland and England. Even so, in our case the parallel processing didn't speed up the calculations. Despite the large RAM available, we were forced to restrict the computations with the parameter set $\left(m, n, N_{\text {min }}\right)=(4,5,80)$ to only $\zeta \leq 40$. At $\zeta=40$ the calculations required two complex-valued matrices of size $48^{\prime} 936 \times 48^{\prime} 936$ using 38 GByte of RAM each, two realvalued matrices of the same size using 19 GByte each, and three complex-valued matrices of size $24^{\prime} 468 \times 24^{\prime} 468$ using 9.5 GByte each, requiring a total of at least 142.5 GByte of RAM. The calculations for the parameter set $\left(m, n, N_{\min }\right)=(4,5,80)$ for all $16 \kappa$-values and for all $\zeta \leq 40$ alone ran for five days and five hours! The computations for $\zeta>40$ were done using the relaxed parameter set $\left(m, n, N_{\min }\right)=(4,3,60)$. At $\zeta=100$ this led to matrices of size $45^{\prime} 184 \times 45^{\prime} 184$. By means of applying a gliding mean between the results obtained with $\left(m, n, N_{\min }\right)=$ $(4,5,80)$ and those obtained with $\left(m, n, N_{\text {min }}\right)=(4,3,60)$, we got very smooth curves over the whole range of $\kappa$ and $\zeta$, see section 3 .

\section{Results and discussion}

With the final parameter sets described in section 2, we obtained results which can be expected to be reasonably precise and which are smooth. Fig. 2 shows the final curves of the ratio $L / L_{\text {skin }}$ in function of $\zeta$ for $\kappa=2.05,3.0$, and 10.0. They are very smooth, and the curve for $\kappa=10.0$ is a horizontal line close to unity, as it should be.

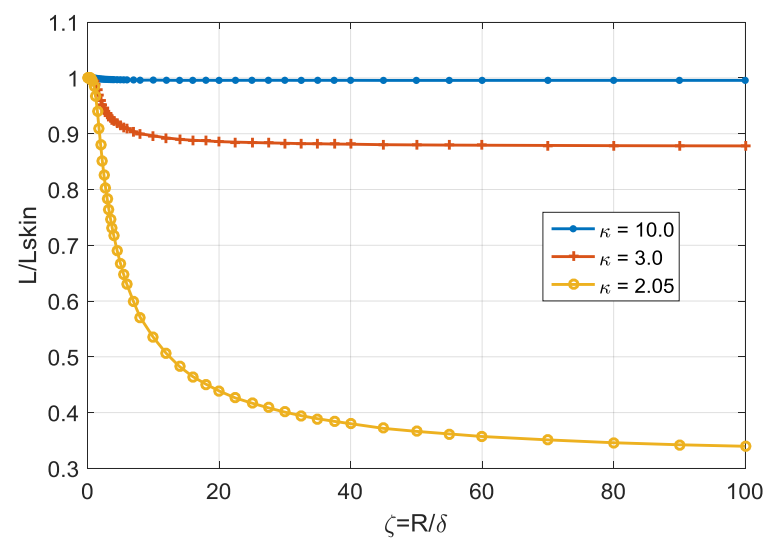

Figure 2: The final ratio $L / L_{\text {skin }}$ in function of $\zeta$ for $\kappa=$ $10.0,3.0$, and 2.05 , in this order from top to bottom.

The results clearly demonstrate the importance of taking the proximity effect into account. Fig. 2 shows that for very close wires, $\kappa=2.05$, i.e. with a gap of $1 / 20$ of a radius between the wires, at $\zeta=100$ the inductance decreases to $34 \%$ of the value it would have without proximity effect. In other words, the inductance calculated considering skin effect only is $(1-0.34) / 0.34 \cdot 100 \% \cong 194 \%$ too large! And even down to much lower $\zeta$ at around $\zeta=10$ the error is still close to $100 \%$ !

At the relative interaxial wire distance $\kappa=3$, i.e. with a gap of one radius between the wires, neglecting the proximity effect leads to an inductance which is still about $14 \%$ too large. The error remains above $10 \%$ for $\zeta$ down to $\zeta=10$. To keep the error in neglecting the proximity effect below $1 \%$, the gap between the wires must encompass at least three wire diameters. Equivalently, the interaxial wire distance must be at least four wire diameters, or $\kappa \geq 8$.

Fig. 3 is a pseudo 3D plot of the complete data for all $\kappa$ and $\zeta$. The surface representing the data is very smooth.

It is easy to calculate the current distribution in the finite filament method ([3], equation (6.73)). Since we have found no figures of the current distribution with proximity effect in the literature, it may be helpful to illustrate it with some examples. Figures $4-7$ show the current distribution normalized to the maximum current when the wires are far apart (upper part of the figures, $\kappa=10$, i.e. with a gap of 4 wire diameters), and close together (lower part of the figures, $\kappa=2.05$, i.e. with a gap of $1 / 40$ wire diameter), at four values $\zeta=0.05,1.0,5.0$, and 40.0 . The magnitude of the current is coded by brightness and color: The scale starts from black for vanishing or non-existing current, 
continuing via brown, dark red, bright red, orange, and yellow up to white for the strongest current.

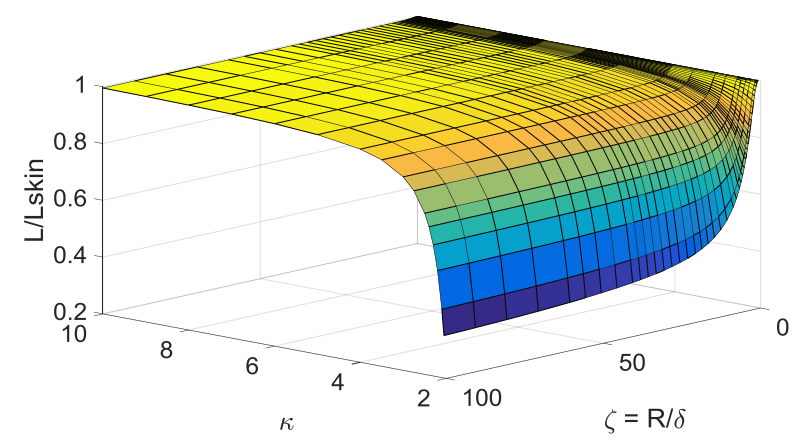

Figure 3: Pseudo 3D plot of the final ratio $L / L_{\text {skin }}$ in function of the dimensionless parameters $\kappa$ and $\zeta$.

Fig. 4 with $\zeta=0.05$ (corresponding to $42.5 \mathrm{~Hz}$ for $R=$ $0.5 \mathrm{~mm}$ ) represents the DC case. The current distribution is uniform both when the wires are far apart and when they are close together: No skin nor proximity effect manifests itself. Fig. 5 shows the normalized current distribution at low $\zeta=$ $1.0(17 \mathrm{kHz}$ for $R=0.5 \mathrm{~mm})$. The current distribution is mostly uniform. There is no skin effect visible at both wire distances, i.e. there is no radial gradient in the current distribution, except in the horizontal direction where the current is slightly stronger on those sides of the wires facing each other. This horizontal gradient is a manifestation of the proximity effect, which already starts to become visible when the wires are far apart. When they move close together, the effect increases. Fig. 6 depicts the situation at moderately high $\zeta=5.0(425 \mathrm{kHz}$ for $R=0.5 \mathrm{~mm})$. Now the skin effect is clearly visible: There is a distinctive radial gradient from the center towards the surface in all directions in the wires. Already when the wires are far apart, the current distribution is clearly asymmetric due to the proximity effect, which tends to concentrate the current towards the near sides of the wires. At close distance the proximity effect strongly increases. The current on the far sides of the wires nearly vanishes. Fig. 7 shows the current distribution at high $\zeta=40(27 \mathrm{MHz}$ for $R=0.5 \mathrm{~mm})$. The effects are now dramatically more pronounced. At close distance the current is exclusively limited to those regions of the wires with the smallest inter-wire distance. Hence, the mutual inductance increases strongly. Due to equation (10) the total inductance decreases dramatically.
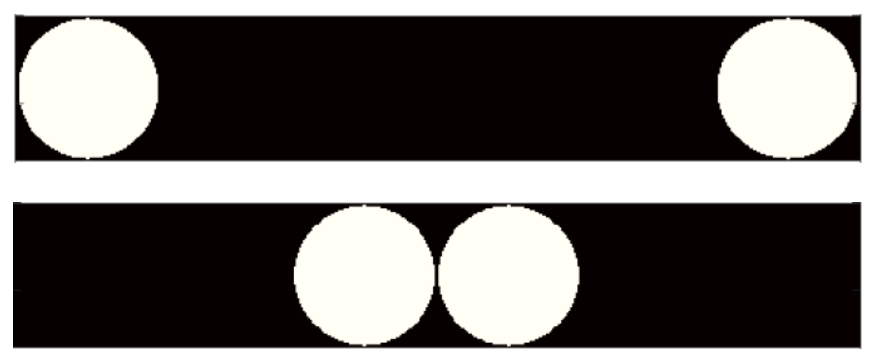

Figure 4: Normalized current distribution at $\zeta=0.05$.
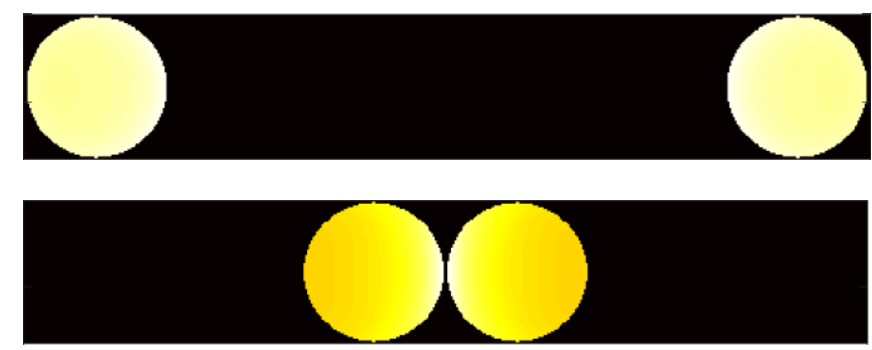

Figure 5: Normalized current distribution at $\zeta=1.0$.
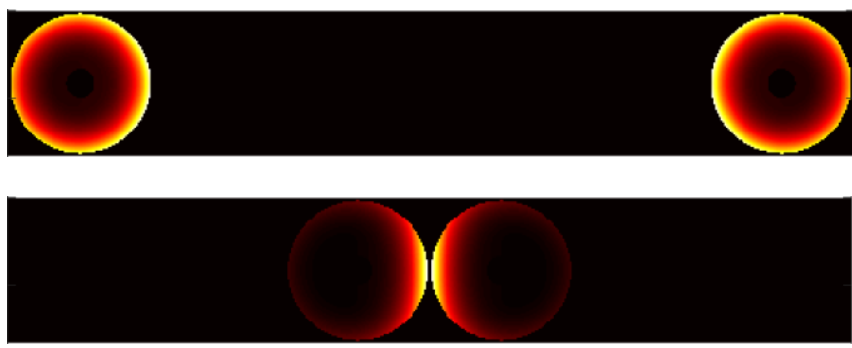

Figure 6: Normalized current distribution at $\zeta=5.0$.
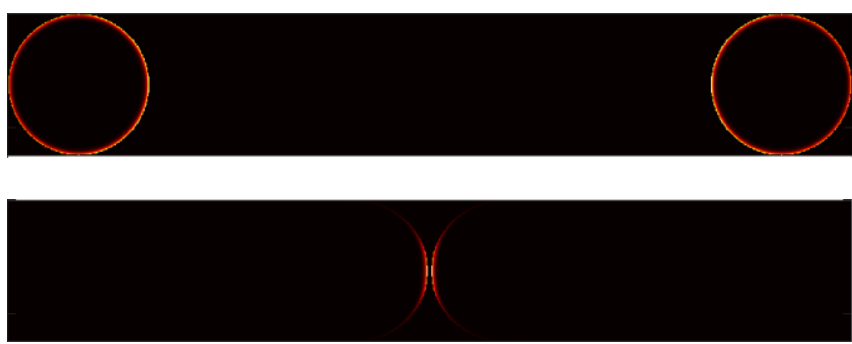

Figure 7: Normalized current distribution at $\zeta=40.0$.

\section{Analytical approximation}

Plots of the internal inductance of single wires with skin effect, normalized to the DC value, in function of $\zeta$ ([12], Fig. 2, where a similar parameter $x$ was used instead of $\zeta$ ) resemble those of the ratio $L / L_{\text {skin }}$ of a two-wire transmission line with proximity effect, see Fig. 2. This suggests that a generalized form of the type of function used there ([12], equations (38) and (57)) might also be applied here. But in [12] it depended only on $\zeta$, whereas here it must also depend on $\kappa$. One way to generalize it to a two-dimensional function is by making its constants functions of $\kappa$. Owing to the relation between the internal inductance and the GMD ([12], equation (12)), we must essentially take the negative logarithm of the expression in curly braces of ([12], equation (57)) to transform it to the internal inductance. Since we deal with a normalized inductance $\left(L / L_{\text {skin }}\right)$, the factor $g_{1}$ of ([12], equation (57)) is irrelevant here. So is the additive constant, which just defined the boundary condition for $\zeta=0$ there. To get the total inductance, we must add a term that does not depend on frequency, and, hence, neither on $\zeta$. But it is expected to depend on $\kappa$. This is the summand $g_{1}(\kappa)$ in the bracket of equation (11). The constant factor $g_{2}$ of $\zeta$ in ([12], equation (57)) is now a function of $\kappa$, $g_{2}(\kappa)$. The exponent of $\zeta$, which was 4 in [12], must now also be made a function of $\kappa$. We designate it by $g_{3}(\kappa)$. The $4^{\text {th }}$ square root of [12] is identical to a power function of 
exponent 0.25 . It may be left constant, but its value must be modified. We have found its optimum value to be 0.3660 by means of a non-linear fit. Further, we have changed the constant 1 prior to the minus sign of ([12], equation (57)) to the value 2 in the large parenthesis of equation (11). The reason is that for $\zeta=0$, the logarithm of the large parenthesis becomes exactly 0 , leaving the summand $g_{1}(\kappa)$ in the bracket. This makes it very easy to guarantee that the ratio $L / L_{\text {skin }}$ is exactly unity for $\zeta=0$ for all $\kappa$, as it must be: We simply divide the bracket of equation (11) by $g_{1}(\kappa)$. This explains the whole appearance of our fit function for the ratio $L / L_{\text {skin }}$, which now reads

$$
\begin{aligned}
\frac{L}{L_{\text {skin }}}(\kappa, \zeta) \cong \frac{1}{g_{1}(\kappa)} & {\left[g_{1}(\kappa)\right.} \\
& -\log (2 \\
& \left.\left.-\frac{1}{\left\{1+\left[g_{2}(\kappa) \cdot \zeta\right]^{g_{3}(\kappa)}\right\}^{0.3660}}\right)\right] .
\end{aligned}
$$

Now, the problem consisted in finding the analytical functions $g_{i}(\kappa), i=1,2,3$. To this end, for each of the 16 values of $\kappa$ enumerated in section 2, we solved an optimizing problem to minimize the maximum deviation of equation (11) in function of $\zeta$ from our reference data of $L / L_{\text {skin }}$ described in section 2. The solution of each of these 16 problems was a triple of values $g_{i}(\kappa), i=1,2,3$ in equation (11) for each value of $\kappa$. Thus, we obtained three smooth curves $g_{i}(\kappa)$, each one given at 16 sampling points $\kappa$. Fitting these three curves, in turn, led to the following functions of $\kappa$, which are defined for $\kappa>2$ and were tested for $\kappa \geq 2.05$,

$$
\begin{aligned}
& g_{1}(\kappa)=\frac{1}{2} \kappa^{2.5}-2, \\
& g_{2}(\kappa)=\frac{1}{16} \log (\kappa-2)+0.5143, \\
& g_{3}(\kappa)=\frac{1}{3} \log (\kappa-2)+3.0532 .
\end{aligned}
$$

The occurrence of the argument $(\kappa-2)$ is well justified because the lower physical limit of $\kappa$ is exactly 2 . Equations (11) - (14) define our analytical approximation. Of course, it can not only be applied to two-wire transmission lines, but also to rectangular coils of closely spaced cylindrical wires, as is done in section 5. In any case, with the help of this analytical approximation, calculating the total inductance with proximity effect is easy:

1. Calculate the normalized interaxial distance $\kappa$ according to equation (1).

2. Calculate $\zeta$ according to equations (2) and (3) with $\sigma$ depending on the conductor material and temperature. For copper use equation (15), $\sigma=1 / \rho$.

3. Evaluate the functions $g_{i}(\kappa), i=1,2,3$ given in equations (12) - (14).

4. Insert these values and $\zeta$ from step 2 into function (11) to get the ratio $L / L_{\text {skin }}$.
5. Calculate the inductance with skin effect only, $L_{\text {skin }}$, in function of the length $l$ with the help of equations (4) - (7).

6. Multiply the ratio $L / L_{\text {skin }}$ from step 4 by $L_{\text {skin }}$ to get the total inductance $L$ of the transmission line.

Example $\left(t_{C}=20^{\circ} \mathrm{C}\right.$. All other quantities are in SI-units): $R=0.5 \cdot 10^{-3} \mathrm{~m}, d=1.025 \cdot 10^{-3} \mathrm{~m}, f=2.72 \cdot 10^{5} \mathrm{~Hz}$, (i.e. $\kappa=2.05, \zeta=4.0$ ) yields $g_{1}=1.0085, g_{2}=0.3271$, $g_{3}=2.0546$, and $L / L_{\text {skin }} \cong 0.7336$ (reference result: $L / L_{\text {skin }}=0.7168$, deviation: $\left.2.3 \%\right)$. For $l=0.5 \mathrm{~m}$ we get $L_{\text {skin }}=1.678 \cdot 10^{-7} \mathrm{H}$, yielding $L=1.231 \cdot 10^{-7} \mathrm{H}$.

To verify the approximation, we computed its maximum deviation from our reference data. The result was $3.0 \%$. It occurred at $\kappa=2.05$ and $\zeta=90$. For $\kappa \geq 3$ the maximum deviation dropped to $0.4 \%$. Fig. 8 depicts a pseudo 3D plot of the percentaged relative deviation of the analytical approximation from the reference data for all $\kappa$ and $\zeta$. It shows that for $\kappa \geq 3$ the deviation is very small.

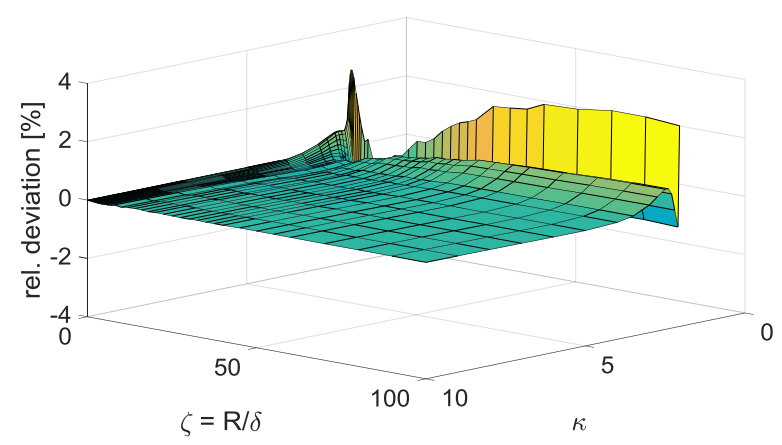

Figure 8: Pseudo 3D plot of the relative deviation of the analytical approximation from the reference data in percent.

\section{Comparison with measurements}

In this section we compare inductances calculated by means of the analytical approximation with results from measurements reported in the literature and from our own measurements.

Kennelly et al. presented results of precision measurements of the total inductance of four very large rectangular loop coils, all the same length $l=27 \mathrm{~m}$, but of different and much smaller widths $d$. They were composed of four cylindrical copper wires of diameter $1.168 \mathrm{~cm}$. The measurements were taken at various frequencies ([5], Table IV). In two of the coils the longer wires were sufficiently close together to be suitable for us to investigate the proximity effect, with gaps of $0.3 \mathrm{~mm}$ and $8 \mathrm{~mm}$, respectively, corresponding to interaxial distances $d=1.198 \mathrm{~cm}$ and $d=$ $1.968 \mathrm{~cm}$, respectively. Since the length $l$ of both coils was more than 1'000 times larger than the width $d$, the two wires of axial length $d$ forming the shorter sides of the rectangular coil could be neglected in calculating the inductance. Hence, we could regard the coils as two-wire transmission lines of length $l$ and interaxial distances $d$, 
corresponding to $\kappa=2.0514$ and $\kappa=3.3699$, respectively. The specific resistance $\rho$ of copper in $\Omega \mathrm{m}$ between $0{ }^{\circ} \mathrm{C}$ and $27^{\circ} \mathrm{C}$ in function of the temperature $t_{C}$ in ${ }^{\circ} \mathrm{C}$ can be well approximated by the polynomial

$$
\rho=10^{-8}\left(2 \cdot 10^{-6} \cdot t_{C}{ }^{2}+0.00671 \cdot t_{C}+1.543\right) \text {. }
$$

It reproduces the tabulated values at $0{ }^{\circ} \mathrm{C}, 20^{\circ} \mathrm{C}$, and $25^{\circ} \mathrm{C}$ exactly, and at $27^{\circ} \mathrm{C}$ the deviation is $+0.036 \%$ ([11], $T=$ $273 \mathrm{~K}, 293 \mathrm{~K}, 298 \mathrm{~K}, 300 \mathrm{~K})$. The measurements for $\kappa=$ 2.0514 were taken at temperatures $t_{C}=21.1^{\circ} \mathrm{C}, 21.4^{\circ} \mathrm{C}$, $21.5^{\circ} \mathrm{C}, 21.5^{\circ} \mathrm{C}, 21.2^{\circ} \mathrm{C}, 21.0^{\circ} \mathrm{C}, 20.9^{\circ} \mathrm{C}, 21.0^{\circ} \mathrm{C}$, and $21.1^{\circ} \mathrm{C}$. They didn't fit into Table 1 . They were considered in the calculations via $\sigma=1 / \rho$ and equations (2), (3), and (15). For $\kappa=3.3699$, no temperatures were given for the first three measurements ([5], Table IV, gap $=8 \mathrm{~mm}$ ). We assumed them to be equal to the temperature of the $4^{\text {th }}$ measurement, $t_{C}=16.3^{\circ} \mathrm{C}$. The temperatures for the remaining measurements, starting from the $5^{\text {th }}$, were $t_{C}=$ $16.5^{\circ} \mathrm{C}, 16.9^{\circ} \mathrm{C}, 17.2^{\circ} \mathrm{C}, 17.8^{\circ} \mathrm{C}, 18.0^{\circ} \mathrm{C}, 18.3^{\circ} \mathrm{C}$, and $18.4^{\circ} \mathrm{C}$. They didn't fit into Table 2 . Tables 1 and 2 show the results of our calculations based on equations (11) (14) compared to those of Kennelly's measurements. The consideration of the temperature changed the deviations in both Tables by only $0.1 \%$ compared to calculations with the temperature fixed to $20^{\circ} \mathrm{C}$.

Table 1: The ratio $L / L_{\text {skin }}$ by equation (11) of a two-wire transmission line at $\kappa=2.0514$, the measured $\left(L_{\text {exp }}\right)$ and calculated $\left(L_{\text {calc }}\right)$ total inductances at various frequencies $f$ and parameters $\zeta$, and the relative deviation of $L_{\text {calc }}$ from $L_{\text {exp }}$.

\begin{tabular}{|c|c|c|c|c|c|}
\hline $\begin{array}{c}f \\
{[\mathrm{~Hz}]}\end{array}$ & $\begin{array}{c}\zeta \\
{[-]}\end{array}$ & $\begin{array}{l}L \\
/ L_{\text {skin }}\end{array}$ & $\begin{array}{c}L_{e x p} \\
{[\mu \mathrm{H}]}\end{array}$ & $\begin{array}{l}L_{\text {calc }} \\
{[\mu \mathrm{H}]}\end{array}$ & $\begin{array}{l}\text { Dev. } \\
{[\%]}\end{array}$ \\
\hline 60 & 0.6923 & 0.9836 & 10.379 & 10.278 & -1.0 \\
\hline 236 & 1.3722 & 0.9400 & 9.851 & 9.740 & -1.1 \\
\hline 740 & 2.4294 & 0.8506 & 8.143 & 8.378 & 2.9 \\
\hline 1000 & 2.8241 & 0.8178 & 7.594 & 7.859 & 3.5 \\
\hline 1473 & 3.4296 & 0.7716 & 6.889 & 7.177 & 4.2 \\
\hline 2038 & 4.0357 & 0.7311 & 6.374 & 6.634 & 4.1 \\
\hline 3058 & 4.9445 & 0.6808 & 5.805 & 6.016 & 3.6 \\
\hline 3918 & 5.5957 & 0.6511 & 5.558 & 5.674 & 2.1 \\
\hline 5170 & 6.4265 & 0.6196 & 5.297 & 5.323 & 0.5 \\
\hline
\end{tabular}

The maximum deviation in Table 1 is $4.2 \%$. In Table 2 it is only $1.1 \%$, reflecting the higher accuracy of the analytic approximation for $\kappa \geq 3$. But Kennelly's measurements are limited to moderately high $\zeta<7$, whereas the largest deviation from the reference data occurred at the considerably higher $\zeta=90$. So, one might suspect to find larger deviations at higher $\zeta$ and at small normalized distances $\kappa<3$.

To find out we conducted our own measurements at high frequencies. For this purpose we had two two-wire transmission lines fabricated by a tinsmith. Each of them consisted of two cylindrical copper rods of diameter $\emptyset=$ $20.0 \mathrm{~mm}$ and equal overall length $l_{\text {tot }}$ mounted in parallel to form the two wires of the transmission line. One end was kept electrically open, and the other was short-circuited. On the open side and in the middle, the long parallel wires were supported by a thin hard rubber disk for increased stability and uniform interaxial distance $d$.

Table 2: The ratio $L / L_{\text {skin }}$ by equation (11) of a two-wire transmission line at $\kappa=3.3699$, the measured $\left(L_{\text {exp }}\right)$ and calculated $\left(L_{\text {calc }}\right)$ total inductances at various frequencies $f$ and parameters $\zeta$, and the relative deviation of $L_{\text {calc }}$ from $L_{\text {exp }}$.

\begin{tabular}{cccccc}
\hline $\begin{array}{c}f \\
{[\mathrm{~Hz}]}\end{array}$ & $\begin{array}{c}\zeta \\
{[-]}\end{array}$ & $\begin{array}{c}L \\
/ L_{\text {skin }}\end{array}$ & $\begin{array}{c}L_{\text {exp }} \\
{[\mu \mathrm{H}]}\end{array}$ & $\begin{array}{c}L_{\text {calc }} \\
{[\mu \mathrm{H}]}\end{array}$ & $\begin{array}{c}\text { Dev. } \\
{[\%]}\end{array}$ \\
\hline 60 & 0.6991 & 0.9981 & 15.894 & 15.777 & -0.7 \\
239 & 1.3953 & 0.9871 & 15.602 & 15.511 & -0.6 \\
671 & 2.3379 & 0.9659 & 14.793 & 14.745 & -0.3 \\
1068 & 2.9495 & 0.9556 & 14.350 & 14.237 & -0.8 \\
1509 & 3.5046 & 0.9489 & 14.007 & 13.880 & -0.9 \\
1991 & 4.0223 & 0.9442 & 13.782 & 13.631 & -1.1 \\
1988 & 4.0168 & 0.9443 & 13.722 & 13.633 & -0.6 \\
2486 & 4.4863 & 0.9410 & 13.560 & 13.458 & -0.7 \\
3028 & 4.9492 & 0.9384 & 13.301 & 13.319 & 0.1 \\
3880 & 5.5990 & 0.9355 & 13.284 & 13.163 & -0.9 \\
4900 & 6.2908 & 0.9332 & 13.127 & 13.034 & -0.7 \\
\hline
\end{tabular}

The short-circuit was realized by a short piece of the same type of rod. It was welded to the parallel long rods at a right angle by means of $45^{\circ}$-slants in both the short rod and the long ones, see Fig. 9. The first transmission line had a gap between the long wires of nominal $4 \mathrm{~mm}$, leading to a nominal interaxial distance $d=2.4 \mathrm{~cm}$, and an overall length $l_{\text {tot }}=60.2 \mathrm{~cm}$. The interaxial distance is given by

$$
d=g a p+2 R
$$

where $R=1.00 \mathrm{~cm}$. The second transmission line had a gap of nominal $8 \mathrm{~mm}$, leading to a nominal interaxial distance of $d=2.8 \mathrm{~cm}$, and an overall length $l_{\text {tot }}=62.45 \mathrm{~cm}$. The effective electric length of the lines was

$$
l=l_{\text {tot }}-R
$$

( $l$ is the length from the open end to the axis of the shorting wire). Thus, we had $l=59.2 \mathrm{~cm}$ and $l=61.45 \mathrm{~cm}$, respectively.

Calculations showed that a deviation of $1 \%$ in the gap between the long wires would result in a deviation in the inductance of less than $0.5 \%$. So, we specified a deviation of roughly $1 \%$ in the gap for the attention of the tinsmith. This was checked on the delivered product with a caliper of $0.01 \mathrm{~mm}$ resolution at six positions evenly distributed along the whole length of the transmission line several times, and a mean value was determined for each position. The results for the first transmission line were $3.91,3.81,3.83,3.86$, 3.86 , and $3.86 \mathrm{~mm}$, respectively. The mean value was 3.86 $\mathrm{mm}$, and the relative standard deviation was $0.9 \%$. This resulted in $d=2.386 \mathrm{~cm}$, and equation (1) yielded $\kappa=$ 2.386. For the second transmission line, the results of the gap measurements were $8.04,7.83,7.80,7.80,7.85$, and $7.95 \mathrm{~mm}$, respectively. 


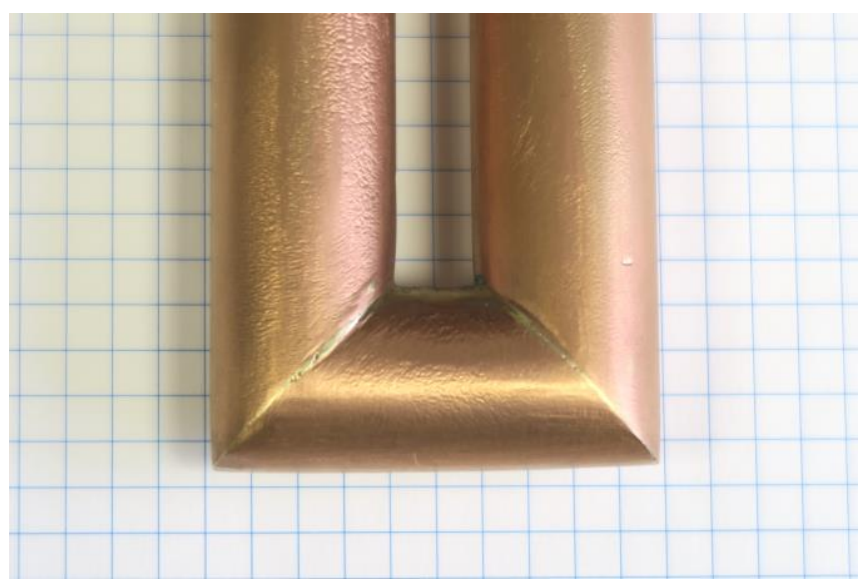

Figure 9: The short-circuited end of the second two-wire transmission line with the slanted welding areas. The gap between the wires is nominally $8 \mathrm{~mm}$, as can be estimated from the grid on the graph paper of spacing $5 \mathrm{~mm}$.

The mean value amounted to $7.88 \mathrm{~mm}$, and the relative standard deviation was $1.2 \%$. This resulted in $d=$ $2.788 \mathrm{~cm}$, and equation (1) yielded $\kappa=2.788$. Consequently, the tinsmith could just about achieve the specified precision of around $1 \%$.

Before doing the measurements, the contacting surfaces were freed from any oxide layer by immersing them in citric acid for a few hours. The temperature was $26^{\circ} \mathrm{C}$. The specific resistance of copper was calculated according to equation (15) and found to be $\rho=1.719 \cdot 10^{-8} \Omega \mathrm{m}$. The resulting specific conductance $\sigma=1 / \rho$ was then used in equation (3).

For these transmission lines, the shorting wire could not be neglected anymore in calculating the inductance, as was possible for Kennelly's coils. We had to take the inductance $L_{d}$ of the shorting wire of axial length $d$ into account by subtracting it from the measured raw inductance $L_{\exp \text { raw }}$ to get the measured inductance $L_{\text {exp }}$ of the two-wire transmission line itself:

$$
L_{\text {exp }}=L_{\text {exp raw }}-L_{d} .
$$

The reason for simply subtracting $L_{d}$ was that the shorting wire was oriented at a right angle to the long parallel wires, so that the mutual inductance between the shorting wire and the long parallel ones vanished.

The inductance of the shorting wire could not simply be calculated with the help of equation (5) by substituting $d$ for $l$, because the requirement $l \gg R$ did not hold anymore $(d / R \cong 2.4$ for the shorting wire, compared to $l / R \cong 60$ for the long parallel wires). Equation (5) is a variant of Wien's formula for arbitrary frequency: It neglects the arithmetic mean square distance AMSD and the arithmetic mean distance AMD of the cross section [14]. For ratios $l / R$ as small as 2.4 , Wien's formula leads to a large error in the order of $-30 \%$ at DC ([14], Fig. 4). In the highfrequency limit, the error even rises to $-44 \%$. For such small ratios $l / R$, a more precise formula for the inductance of the shorting wire must be used, namely ([14], equation (33))

$$
\begin{gathered}
L_{d}=\frac{\mu_{0}}{2 \pi}\left[d \cdot \log \left(\sqrt{d^{2}+A M S D^{2}}+d\right)-d \cdot \log G M D\right. \\
\left.-\sqrt{d^{2}+A M S D^{2}}+A M D\right],
\end{gathered}
$$

where we have substituted $d$ for $l$. At high frequencies the inductance can be well approximated by its high frequency limit. It is obtained by substituting the high-frequency limits for the mean distances in equation (17), which are

$$
\begin{gathered}
G M D=R, \\
A M S D=\sqrt{2} R, \\
A M D=\frac{4}{\pi} R
\end{gathered}
$$

([14], equations (21), (24), and (26), respectively). $L_{d}$ calculated this way neither depends on temperature, nor on frequency. We got $L_{d}=4.8 \mathrm{nH}(\kappa=2.386)$ and $L_{d}=6.2 \mathrm{nH}$ $(\kappa=2.788)$, respectively.

The measurements of $L_{\text {exp raw }}$ were performed with an Agilent $^{\mathbb{R}}$ 4294A Precision Impedance Analyzer with a 42941A Impedance Probe. According to Fig. 10-6 in the user manual, the accuracy of this set-up for inductances around $150 \mathrm{nH}$ was $3 \%$ at $20 \mathrm{kHz}$ and $1 \%$ at $10 \mathrm{MHz}$. For measuring such small impedances (i.e. $(0.4+19.4 i) \mathrm{m} \Omega$ at $20 \mathrm{kHz}$ for $\kappa=2.386$ ), it was important to use a massive piece of copper for the shorting calibration. We used a circular disk of diameter $30 \mathrm{~mm}$ and thickness $12 \mathrm{~mm}$. Its contacting surface had also been cleaned with citric acid prior to calibration. The values $L_{\text {exp raw }}$ were corrected for $L_{d}$ by means of equation (16) with the values for $L_{d}$ given above.

The results are presented in Table 3 for $\kappa=2.386$ and Table 4 for $\kappa=2.788$. Assuming the standard temperature of $20^{\circ} \mathrm{C}$ instead of $26^{\circ} \mathrm{C}$ would change the deviations in both Tables by less than $0.1 \%$.

The limit $\zeta=100$ in the reference data was clearly exceeded in most of the measurements. But even so, the results from the analytical approximation remained precise. In more usual wires, i.e. in ones of smaller diameter than in our experiment, like e.g. $\emptyset=1 \mathrm{~mm}, \zeta=479$ corresponds to a frequency of $4 \mathrm{GHz}$.

Tables 3 and 4 confirm the validity of our approximation also for large $\zeta$. The maximum deviation of $2.4 \%$ from Table 4 lies below the one of $4.2 \%$ from Table 1 . Thus, our initial apprehension that at higher $\zeta$ there might be larger deviations has been dispelled.

Furthermore, Fig. 10 demonstrates that the two-dimensional function defined by equations (11) - (14) even extrapolates smoothly and plausibly up to infinity in both $\kappa$ and $\zeta$.

Nevertheless, the measured results $L_{\text {exp }}$ in Tables 3 and 4 seem to contradict a continuous decrease of the inductance for high enough frequencies, since, in contrast to the calcu- 
lated values $L_{\text {calc }}$, the measured ones $L_{\text {exp }}$ seem to increase again slightly but continuously beyond $3-5 \mathrm{MHz}$.

Table 3: The ratio $L / L_{\text {skin }}$ by equation (11) of a two-wire transmission line at $\kappa=2.386$, the measured $\left(L_{\text {exp }}\right)$ and analytically approximated $\left(L_{\text {calc }}\right)$ inductance at various frequencies $f$ and parameters $\zeta$, and the relative deviation of $L_{\text {calc }}$ from $L_{\text {exp }}$.

\begin{tabular}{cccccc}
\hline$f$ & $\zeta$ & $L$ & $L_{\text {exp }}$ & $\begin{array}{c}L_{\text {calc }} \\
{[\mathrm{nH}]}\end{array}$ & $\begin{array}{c}\text { Dev. } \\
{[\%]}\end{array}$ \\
\hline $\mathrm{MHz}]$ & {$[-]$} & $/ L_{\text {skin }}$ & {$[\mathrm{nH}]$} & {$[43.0$} & -1.1 \\
0.02 & 21.433 & 0.7327 & 149.7 & 148.0 & -0.9 \\
0.05 & 33.888 & 0.7245 & 146.3 & 144.9 & -0.9 \\
0.075 & 41.505 & 0.7220 & 143.9 & 143.9 & 0.0 \\
0.1 & 47.925 & 0.7205 & 143.7 & 143.3 & -0.2 \\
0.2 & 67.777 & 0.7176 & 143.5 & 142.2 & -0.9 \\
0.5 & 107.16 & 0.7151 & 141.8 & 141.3 & -0.3 \\
1.0 & 151.55 & 0.7138 & 141.0 & 140.8 & -0.1 \\
2.0 & 214.33 & 0.7129 & 140.6 & 140.5 & -0.1 \\
3.0 & 262.50 & 0.7126 & 140.4 & 140.3 & 0.0 \\
5.0 & 338.88 & 0.7122 & 140.2 & 140.2 & 0.0 \\
7.0 & 400.97 & 0.7120 & 140.6 & 140.1 & -0.3 \\
8.0 & 428.66 & 0.7119 & 140.8 & 140.1 & -0.5 \\
9.0 & 454.66 & 0.7118 & 141.0 & 140.0 & -0.7 \\
10.0 & 479.25 & 0.7118 & 141.2 & 140.0 & -0.8 \\
\hline
\end{tabular}

Table 4: The ratio $L / L_{\text {skin }}$ by equation (11) of a two-wire transmission line at $\kappa=2.788$, the measured $\left(L_{\text {exp }}\right)$ and analytically approximated $\left(L_{\text {calc }}\right)$ inductance at various frequencies $f$ and parameters $\zeta$, and the relative deviation of $L_{\text {calc }}$ from $L_{\text {exp }}$.

\begin{tabular}{|c|c|c|c|c|c|}
\hline $\begin{array}{c}f \\
{[\mathrm{MHz}]}\end{array}$ & $\begin{array}{c}\zeta \\
{[-]}\end{array}$ & $\begin{array}{l}L \\
/ L_{\text {skin }}\end{array}$ & $\begin{array}{l}L_{\text {exp }} \\
{[\mathrm{nH}]}\end{array}$ & $\begin{array}{l}L_{\text {calc }} \\
{[\mathrm{nH}]}\end{array}$ & $\begin{array}{l}\text { Dev. } \\
{[\%]}\end{array}$ \\
\hline 0.02 & 21.433 & 0.8542 & 215.2 & 210.8 & -2.1 \\
\hline 0.05 & 33.888 & 0.8508 & 211.9 & 208.1 & -1.8 \\
\hline 0.075 & 41.505 & 0.8498 & 210.4 & 207.3 & -1.5 \\
\hline 0.1 & 47.925 & 0.8491 & 209.3 & 206.8 & -1.2 \\
\hline 0.2 & 67.777 & 0.8480 & 208.7 & 205.9 & -1.3 \\
\hline 0.5 & 107.16 & 0.8471 & 207.7 & 205.1 & -1.2 \\
\hline 1.0 & 151.55 & 0.8466 & 207.0 & 204.7 & -1.1 \\
\hline 2.0 & 214.33 & 0.8463 & 206.8 & 204.4 & -1.1 \\
\hline 3.0 & 262.50 & 0.8462 & 206.7 & 204.3 & -1.1 \\
\hline 5.0 & 338.88 & 0.8460 & 206.9 & 204.2 & -1.3 \\
\hline 7.0 & 400.97 & 0.8460 & 207.5 & 204.1 & -1.6 \\
\hline 8.0 & 428.66 & 0.8459 & 207.9 & 204.1 & -1.8 \\
\hline 9.0 & 454.66 & 0.8459 & 208.4 & 204.1 & -2.1 \\
\hline 10.0 & 479.25 & 0.8459 & 209.0 & 204.1 & -2.4 \\
\hline
\end{tabular}

We suspected that this might be the effect of a measuring artifact caused by a resonance somewhere far beyond $10 \mathrm{MHz}$. Indeed, letting the Impedance Analyzer scan beyond $10 \mathrm{MHz}$ revealed that the measured inductance seemed to continue rising with ever increasing slope, finally culminating in a maximum at the resonance frequency. In the first transmission line $(\kappa=2.386)$, the resonance was observed at $81.3 \mathrm{MHz}$, and in the second one $(\kappa=2.788)$ at $79.9 \mathrm{MHz}$. Knowing the capacitance allows to calculate the resonance frequency $f_{\text {res }}=1 /\left(2 \pi \sqrt{L_{\text {tot }} C}\right)$, compare it to the measured values and maybe confirm that the artifact was indeed due to $\mathrm{LC}$ resonance.

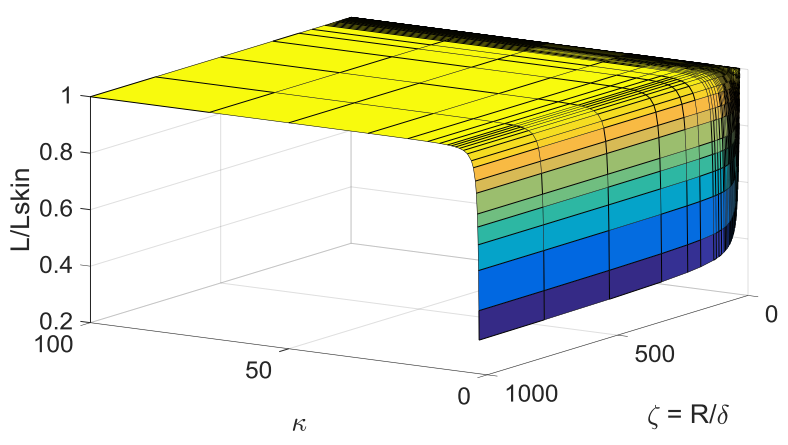

Figure 10: Pseudo 3D plot of the analytically approximated ratio $L / L_{\text {skin }}$ for very large $\kappa$ and $\zeta$.

We had to add the inductance $L_{d}$ of the shorting wire to $L$ because the measurements were always done on shorted transmission lines: $L_{t o t}=L+L_{d}$. The capacitance of two parallel cylinders in the vacuum is given by ([16], equation (12.42), thereby correcting a typing error)

$$
C=\frac{\pi \varepsilon_{0} l}{\log \left(\frac{d}{2 R}+\sqrt{\left(\frac{d}{2 R}\right)^{2}-1}\right)}
$$

where $\varepsilon_{0} \approx 8.8542 \cdot 10^{-12} \mathrm{As} /(\mathrm{Vm})$ is the vacuum permittivity. For the first transmission line $(\kappa=2.386)$ this yielded $C=26.9 \mathrm{pF}$. Together with an inductance $L_{\text {tot }}=$ $(140+4.8) n H$ the calculated resonance frequency was $f_{\text {res }}=80.6 \mathrm{MHz}$. For the second transmission line $(\kappa=$ $2.788)$ we got $C=19.8 \mathrm{pF}, L_{t o t}=(204+6.2) \mathrm{nH}$, and $f_{\text {res }}=78.0 \mathrm{MHz}$. The calculated resonance frequencies agreed to $-0.9 \%$ and $-2.4 \%$, respectively, with the measured ones. This confirmed the suspected nature of the resonance.

Using equation (5) for calculating $L_{d}$ or, equivalently, neglecting the AMSD and AMD in equation (17), would yield $L_{d}=2.7 \mathrm{nH}(\kappa=2.386)$ and $L_{d}=4.0 \mathrm{nH}(\kappa=2.788)$, respectively. The inductances would be $44 \%$ and $35 \%$ too small, respectively. Consequently, the deviations in Table 3 would all worsen by $1.39-1.50 \%$, and those in Table 4 by $0.99-1.04 \%$. Equation (17) for calculating the inductance of short wires as published earlier ([14], equation (33)) was never tested experimentally. Our result of consistent worsening of $L_{\text {calc }}$ when $L_{d}$ is not calculated according to the full equation (17) represents a first tentative and indirect experimental confirmation of this equation.

By the way, equations (11) - (14) for $L$ and equation (18) for $C$ allow to compute the precise wave impedance of twowire transmission lines, even in function of the frequency, via the equation $Z_{\text {wave }}=\sqrt{L / C}$. 


\section{Conclusions}

It seems that not much information is available on the inductance of cylindrical two-wire transmission lines in the literature, and that its calculation requires extensive numerical computations.

In this paper we have produced systematic data of the inductance of cylindrical two-wire transmission lines with proximity effect at various wire distances and frequencies by means of the finite filament method. To achieve sufficient accuracy, very large systems of linear equations had to be solved. As a result, we were forced to deploy the calculations to an external computer with very large RAM.

We have shown that the proximity effect cannot be neglected in inductance calculations unless the interaxial distance between the wires amounts to at least four wire diameters. We have further presented figures of the current distribution in various representative situations, and we have discussed them in detail.

The main aim of the paper has been to develop a simple but precise analytical approximation for the inductance of cylindrical two-wire transmission lines and rectangular coils of closely spaced cylindrical wires of any size taking the proximity effect into account. To this end we have used our data of numerically computed inductances as a reference for constructing a two-dimensional fit with elementary functions. The comparison of the inductances obtained from the analytical approximation with measured results has confirmed its precision up to very high frequencies $(\zeta=480$ or $f=4 \mathrm{GHz}$ for wires of $\emptyset=1 \mathrm{~mm}$ ). Our analytical approximation dramatically reduces both the time and storage capacity needed to calculate the inductance of two-wire transmission lines considering the proximity effect. For all 768 parameter combinations of our reference data, the reduction is from one week on an Intel Xeon processor operating at $2.3 \mathrm{GHz}$ with 256 GByte RAM in the Amazon EC2 cloud to 0.01 seconds and a few kByte on a usual PC.

\section{Acknowledgements}

The first author wishes to thank LEGIC Identsystems AG for granting the time to work on this project, and Mr. G. Ruinelly from Schleh AG, Switzerland, for fabricating the precision two-wire transmission lines. The help of MathWorks Switzerland and England in using Amazon's cloud computers is also highly acknowledged.

\section{References}

[1] A. Sommerfeld, Elektrodynamik, Verlag Harry Deutsch, Frankfurt am Main, 1988, $4^{\text {th }}$ ed. 2001.

[2] L. D. Landau and E. M. Lifschitz, Elektrodynamik der Kontinua, Akademie-Verlag Berlin, Berlin, $5^{\text {th }}$ ed. 1985, first published by edition NAUKA, Moscow, 1982 .

[3] C. R. Paul, Inductance, John Wiley \& Sons, Hoboken, NJ, 2010 .
[4] G.S. Smith, A Simple Derivation for the Skin Effect in a Round Wire, European Journal of Physics, vol. 35, $1-13,2014$

[5] A. E. Kennelly, F. A. Laws, and P. H. Pierce, Experimental Researches on Skin Effect in Conductors, Transactions of the American Institute of Electrical Engineers, vol. XXXIV, no. 2, 1953-2021, 1915.

[6] D. Lovrić, V. Boras, and S. Vujević, Accuracy of Approximate Formulas for Internal Impedance of Tubular Cylindrical Conductors for Large Parameters, Progress in Electromagnetics Research M, vol. 16, 171-184, 2011.

[7] M. J. Tsuk and J. A. Kong, A Hybrid Method for the Calculation of the Resistance and Inductance of Transmission Lines with Arbitrary Cross Sections, IEEE Transactions on Microwave Theory and Techniques, vol. 39, no. 8, 1338-1347, 1991.

[8] J. R. Carson, LIV. Wave propagation over parallel wires: The proximity effect, Philosophical Magazine Series 6, vol. 41, no. 244, 607-633, 1921.

[9] B.-Z. Wang, Exact Expressions for the AC Resistance and Internal Inductance of a Lossy Circular Two-Wire Transmission Line, Microwave and Optical Technology Letters, vol. 7, no. 10, 451-454, 1994.

[10] W. T. Weeks, L. L. Wu, M. F. McAllister, and A. Singh, Resistive and Inductive Skin Effect in Rectangular Conductors, IBM Journal of Research and Development, vol. 23, no. 6, 652-660, 1979.

[11] W. M. Haynes, Th. J. Bruno, and D. R. Lide, CRC Handbook of Chemistry and Physics, 95 ${ }^{\text {th }}$ ed., CRC Press, Taylor \& Francis Group, Boca Raton, FL, p. 1241, 2014.

[12] H. A. Aebischer and B. Aebischer, The GMD Method for Inductance Calculation Applied to Conductors with Skin Effect, Advanced Electromagnetics, vol. 6, no. 2, 77-92, 2017, DOI: http://dx.doi.org/10.7716/aem.v6i2.464.

[13]E. B. Rosa, The Self and Mutual Inductances of Linear Conductors, Bulletin of the Bureau of Standards, vol. 4, no. 2, 301-344, 1907.

[14]H. A. Aebischer and B. Aebischer, Improved Formulae for the Inductance of Straight Wires, Advanced Electromagnetics, vol. 3, no. 1, 31-43, 2014, DOI: http://dx.doi.org/10.7716/aem.v3i1.254

[15]R. Michaely, The MathWorks GmbH, Bern, Switzerland, private communication.

[16]K. Kupfmüller, W. Mathis, and A. Reibiger, Theoretische Elektrotechnik, Springer-Verlag, Berlin, $17^{\text {th }}$ ed., 2006. 